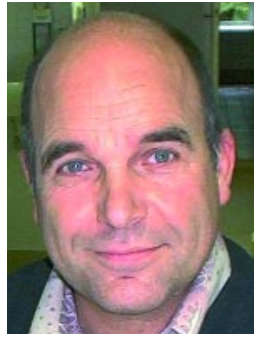

Heinz Hättig

\title{
Neuropsychologische Aspekte der Epilepsien
}

\author{
Heinz Hättig \\ Epilepsie-Zentrum Berlin-Brandenburg, im Verbund der v. Bodelschwinghschen Anstalten Bethel, \\ Evangelisches Krankenhaus Königin Elisabeth Herzberge, Akademisches Lehrkrankenhaus der \\ Charité Berlin
}

psychoneuro 2004; 30 (2): 95-100

Ü ber lange historische Perioden hinweg waren auffällige iktuale und interiktuale Verhaltensäußerungen eines EpilepsiePatienten nicht zu verstehen. Sie wurden seiner Persönlichkeit oder einem Dämon in ihm zugeschrieben und führten häufig zu einer entsprechenden Stigmatisierung des Betroffenen. Erst mit dem zunehmenden Wissen über lokalisierte Hirnfunktionen und der elektrophysiologischen Beschreibung der Hirnaktivität durch das EEG wurde es möglich, die beobachtbaren Verhaltensänderungen als neuropsychologische Störungen zu interpretieren.

Während früher der Patient durch globale Wesens-, Charakteroder Temperaments-Typologien beschrieben wurde, erfolgt dies heute durch ein Profil einzelner neuropsychologischer Funktionen. Kognitive, psychosoziale und psychiatrische Aspekte werden dabei getrennt berücksichtigt. Unter neuropsychologischen Funktionen sind abgrenzbare und selektiv störbare psychische Grundfunktionen zu verstehen, auf die sich das individuelle geistige Leben stützt. Hierzu gehören Verhaltensbereiche wie Gedächtnis, Auf-

Epilepsie-Patienten müssen neben den wiederkehrenden Anfällen häufig auch Störungen ihrer kognitiven Funktionen hinnehmen. Einzelne Patienten können dies als wesentlich einschränkender erleben als den Anfall selbst. Meist klagen die Patienten über eine allgemeine mentale Verlangsamung, über Schwierigkeiten bei Aufgaben, die eine komplexe Informationsverarbeitung erfordern, über Gedächtnisstörungen sowie über Störungen der Aufmerksamkeit und Konzentration. Eine Vielzahl von epileptologischen Faktoren bestimmen im Einzelfall das Profil der kognitiven Störungen. Die klinische Neuropsychologie trägt dazu bei, die kognitiven Funktionen des Patienten zu erfassen, zu bewahren oder sogar zu verbessern. Sie macht EpilepsieBehandlungen sicherer und ist inzwischen zu einem unverzichtbaren Instrument geworden, um die Ergebnisqualität einer Behandlung zu kontrollieren.

merksamkeit, Wahrnehmung, Sprache oder das Problem-Löse-Verhalten. Neben den kognitiven Leistungsmerkmalen zählen aber auch psychische Aspekte, wie Antrieb oder Gestimmtheit dazu. Mit einer Epilepsie gehen häufig Störungen dieser kognitiven Funktionen einher, die durch einen Komplex aus mehreren interagierenden Faktoren verursacht werden (Tab. 1).

\section{Neuropsychologie} epileptischer Syndrome

Aus der Kenntnis des epileptischen Syndroms eines Patienten lassen sich kaum mehr als allgemeine Vermutungen über seinen kognitiven Zustand ableiten. Einen engeren Zusammenhang mit den aktuellen kognitiven Leistungsmöglichkeiten haben dagegen Faktoren, die mittelbar mit den Syndromen verknüpft sind wie die Schwere und die Frequenz der Anfälle. Sie modulieren kurz und mittelfristig die kognitiven Funktionen auf einem Hintergrund überdauernder kognitiver Veränderungen, die wiederum durch die Ätiologie und die Lokalisation einer fokalen Epilepsie oder durch das Alter bei Epilepsiebeginn bestimmt werden. Nur wenige Studien haben sich daher mit den Effekten der epileptischen Syndrome auf die Kognition beschäftigt, die meisten davon untersuchten erwachsene Patienten mit fokalen Epilepsien (7).

Das Vorliegen einer lokalisierten morphologischen oder funktionel- 


\section{Tab. 1 Epileptologische Einflussfaktoren auf die neuropsychologischen Funktionen \\ Ätiologie \\ Epilepsie, Syndrom \\ Anfallstyp \\ Anfallsfrequenz \\ Medikamente \\ Lebensabschnitt \\ Krankheitsentwicklung \\ idopathisch, traumatisch, hypoxisch, u.a. \\ fokal (Lokalisation, Seite, Region), idiopathisch generalisiert \\ Absence, fokale Anfälle, gen. Anfälle, Status Epilepticus \\ selten, häufig, wöchentlich, täglich \\ spez. Nebenwirkungen der Antiepileptika, Sedierung, Aktivierung \\ Alter bei Epilepsiebeginn, Dauer der Epilepsie \\ Progression, Therapieresistenz, benigner Verlauf}

len Läsion kommt den neuropsychologischen Konzepten über lokalisierte Hirnfunktionen entgegen und ihre Effekte können mit einem ähnlichen Methodeninventar untersucht werden wie bei Patienten mit Hirnläsionen aufgrund von Infarkten oder Tumoren.

\section{Fokale Epilepsien}

Den fokalen Epilepsien im Erwachsenenalter liegen überwiegend erworbene Hirnschädigungen zu Grunde, in deren Nähe die Anfälle häufig ihren Ursprung haben. Die Lokalisation sowohl der strukturellen als auch der funktionellen Schädigung beeinflusst das kognitive Leistungsprofil. Durch bestimmte Ausbreitungswege der Anfälle kann sich der Schwerpunkt der funktionellen Läsion jedoch deutlich vom Ort der strukturellen Läsion entfernen, so dass die beobachteten neuropsychologischen Defizite manchmal weniger prägnant oder untypisch für die Lokalisation der strukturellen Läsion erscheinen.

Die häufigste Form einer fokalen Epilepsie ist die Temporal-LappenEpilepsie (TLE), die zu 70\% mit einer Hippokampussklerose assoziiert ist und dann als mesiale TLE (MTLE) bezeichnet wird. Extra-hippokampale TLE gehen meist auf Dysplasien und Tumore zurück. Da dabei meistens eine umschriebene strukturelle Schädigung vorliegt und weil sie sehr häufig vorkommt, wurde die MTLE zum am besten untersuchten epileptischen Syndrom (7). Die neuropsychologischen Hauptsymptome einer MTLE sind Störungen in der Langzeitgedächtnisbildung im verbalen oder non-verbalen Bereich, je nachdem, ob der Hippokampus der sprachdominanten Hemisphäre betroffen ist oder nicht. Von Langzeitgedächtnis (LZG) spricht man schon, wenn das Behaltensintervall länger als eine Minute ist. Bereits an der Stabilisierung von Gedächtnisinhalten über wenige Minuten ist der Hippokampus maßgeblich beteiligt. Dabei kann bei den Patienten das Kurzzeit- oder Arbeitsgedächtnis (KZG) mit einem Behaltensintervall von bis zu 30 Sekunden normal sein. Beide Gedächtnissysteme können selektiv betroffen sein, weil sie durch unterschiedliche biologische Prozesse vollzogen werden. Bei der LZG-Bildung wird letztlich durch eine hippokampale Funktion eine Proteinsynthese initialisiert, die zum Auf- oder Abbau von synaptischer Kontaktfläche führt, während das KZG durch reversible Vesikel-Bereitstellungen an den Synapsen realisiert wird.

Ist der Hippokampus der sprachdominanten Hemisphäre betroffen, so hat der Patient Schwierigkeiten, neue sprachliche Inhalte überdauernd zu stabilisieren. Auch der Abruf von bereits etablierten sprachlichen Inhalten kann betroffen sein. Anzeichen hierfür sind z.B. Wortfindungsstörungen, die vermutlich auf die Ausbreitung der epileptischen Aktivität in temporolaterale Kortexareale zurückzuführen sind. Daneben besteht meist eine moderate Reduktion der Intelligenzfunktionen, der Sprachfunktionen, der visuo-spatialen Funktionen sowie des Schulerfolges. Gleichzeitig können bei diesen Patienten aber die wichtigsten Frontallappenfunktionen wie das KZG, die Aufmerksamkeitsfunktionen oder das Problem-Löseverhalten normal sein.

Bereits ohne sekundäre Generalisierung der Anfälle wurde in mehreren PET-Studien bei einer TLE auch ein frontaler Hypometabolismus be- obachtet. Eine sekundäre Generalisierung der Anfälle erhöht das Risiko für kognitive Dysfunktionen. In diesen Fällen kann es zu umfassenderen intellektuellen Einbußen und zu Störungen der spezifischen präfrontalen Funktionen kommen. Obwohl der Grad einer Demenz selten erreicht wird, schneiden Patienten mit einer längeren Laufzeit der Epilepsie (über 30 Jahre) in Intelligenztests schlechter ab als Patienten mit kürzeren Laufzeiten.

Die Frontallappenepilepsie (FLE) ist nach der TLE die zweithäufigste Epilepsie. Je nach Lokalisation der epileptogenen Zone können Anfälle nur mit Muskelzuckungen (einfache fokale klonische Anfälle aus dem primär motorischen Kortex), tonische Anfälle (aus dem supplementär-motorischen Areal) oder komplex-partielle Anfälle mit Bewusstseinsstörungen, aus den übrigen präfrontalen Regionen resultieren (fronto-orbital, -dorsal, -lateral, -mesial, -polar). Die neuropsychologischen Defizite zeigen sich meist in einer Reduktion des psychomotorischen Tempos und in einer verkürzten Aufmerksamkeitsspanne sowie in einer verschlechterten Merkfähigkeit. In einzelnen Fällen können zusätzlich Störungen in der allgemeinen Verhaltenssteuerung vorliegen.

Fokale Epilepsien aus dem Parietal- und Okzipitallappen sind dagegen vergleichsweise selten, so dass zu diesen Syndromen bisher keine umfassenden neuropsychologischen Untersuchungen vorliegen. Ihre Anfälle neigen dazu, sich in den Frontallappen auszubreiten. Den Epilepsien aus dem Parietalund Okzipitallappen liegen häufig Schädel-Hirn-Traumata, kortikale Entwicklungsstörungen und Tumore als Ursachen zu Grunde. Die Symptome, die bei einer Epilepsie des Parietallappens im Rahmen einer Aura vorkommen, sind vielgestaltig und können sich in Parästhesien, Dysästhesien, Schmerzerlebnissen, sexuellen Empfindungen und in Körperschemastörungen äußern. Die neuropsychologischen Symptome variieren dabei beträchtlich in Abhängigkeit von der Lateralisation, der Lokalisation, 
vom Alter der Läsion und vom Alter beim Beginn der Epilepsie. Im Einzelfall können daraus visuelle Benennungsstörungen (Agnosien), Neglect-Symptome, visuo-spatiale und visuo-konstruktive Störungen sowie Apraxien und linguistische Störungen hervorgehen.

Bei einer okzipitalen Epilepsie treten als Aura meist elementare visuelle (Pseudo-) Halluzinationen im Gesichtsfeld auf. Als iktale Phänomene können Blindheit, AugenDeviation, Augen-Bewegungs-Empfindungen sowie nystagmus-artige Bewegungen vorkommen. Den Patienten mit einer Okzipitallappenepilepsie lässt sich darüber hinaus kein überdauerndes charakteristisches neuropsychologisches Leistungsprofil zuordnen.

\section{Idiopathisch generalisierte Epilepsien (IGE)}

Generalisierte tonisch-klonische Anfälle wirken sich nachteiliger auf die kognitiven Funktionen aus als einfache oder komplex-partiale Anfälle. Bei den idiopathisch generalisierten Epilepsien verdichten sich die Hinweise dafür, dass der Frontallappen die Ursprungsregion der Anfälle darstellt. In Übereinstimmung mit den elektrophysiologischen Befunden sind auch die neuropsychologischen präfrontalen Funktionen wie das Arbeitsgedächtnis oder die Umstellfähigkeit betroffen, die jedoch für die IGE nicht pathognomisch sind, da auch bei TLE ähnliche Funktionsstörungen beobachtet werden können. Die neuropsychologischen Defizite bei IGE werden in einen ursächlichen Zusammenhang mit metabolischen und morphologischen Veränderungen des Frontallappens gebracht.

\section{Neuropsychologie in der Epilepsiechirurgie}

Die neuropsychologischen Aufgaben in der Epilepsiechirurgie liegen zunächst in der Erhebung eines kognitiven Leistungsprofils und der Erfassung der vorliegenden Sprachlateralisation. Diese Befunde werden dann in eine Beziehung gesetzt zur Lokalisation des Anfallsurprunges und gegebenenfalls zu den vorliegenden strukturellen Läsionen.
Hieraus versucht man abzuschätzen, welche kognitiven Restfunktionen das zu resizierende Gewebe noch hat und welche kompensativen Ressourcen dem Patienten zur Verfügung stehen. Diese Überlegungen münden schließlich in eine Prognose über die zu erwartende weitere kognitive Entwicklung nach der Operation. Die Neuropsychologie ist somit ein wesentlicher Teil der präund post-operativen Untersuchungen bei epilepsiechirurgischen Patienten. In dem sie Informationen über die epilepsie-bezogenen kognitiven Störungen erhebt, kann sie dazu beitragen, die Epilepsie zu lateralisieren und zu lokalisieren. Die klinische Neuropsychologie kann die kognitiven Funktionen des Patienten bewahren oder sogar verbessern. Sie macht die Epilepsiechirurgie sicherer und ist inzwischen zu einem unverzichtbaren Instrument geworden, um die Ergebnisqualität - auch über die Frage nach Anfallsfreiheit hinaus - zu kontrollieren (6).

Eine prächirurgische neuropsychologische Basis-Untersuchung dauert meist drei bis vier Stunden und hat ihren Schwerpunkt im Bereich der Gedächtnisfunktionen. Sie wird durch weitere Tests an die individuellen Störungsbereiche des Patienten angepasst und kann durch zusätzliche experimentelle Untersuchungen vervollständigt werden. Bei der Basis-Untersuchung besteht zwischen den verschiedenen Zentren weitgehende Übereinstimmung hinsichtlich der Testverfahren, die zum Einsatz kommen sollten. Die invasiven und experimentellen Prozeduren unterscheiden sich in den verschiedenen Zentren jedoch stärker und unterliegen auch einem Wandel durch die medizinisch-technische Entwicklung. Dies zeigt sich z.B. an der veränderten Indikationsstellung für den Wada-Test. Beim Wada-Test wird über einen Katheter ein Betäubungsmittel in die carotis interna injiziert und für wenige Minuten im entsprechenden Versorgungsgebiet eine Inaktivierung ausgelöst. Aus der Sprachfähigkeit während der Inaktivierung können Rückschlüsse auf die individuelle Sprachlateralisierung gezogen werden. Zunehmend wird jedoch heute zur Sprach- und Ge- dächtnislokalisation auf eine fMRT Untersuchung zurückgegriffen (6).

\section{Zeitlicher Abstand zwischen Anfall und Untersuchung}

Manche Autoren empfehlen, dass die neuropsychologischen Untersuchungen möglichst erst zwölf oder mehr Stunden nach einem komplexpartialen Temporal- oder Frontallappenanfall stattfinden sollten. Andererseits gibt es Untersuchungen, die zeigen, dass Patienten in den Tests schon drei Stunden nach einem Anfall ihre Ausgangsleistung wieder erreichen können. Gegen eine solche rasche und vollständige Leistungserholung sprechen jedoch die immer wieder beobachteten kumulierenden Effekte von dichten Anfällen. Die Diskrepanz ist vermutlich darin begründet, dass die Leistung in einem einzelnen Test einen ganzen Funktionsbereich nicht völlig repräsentiert. Darüber hinaus wurden die Tests nicht dafür konstruiert, länger anhaltende, durch Anfälle ausgelöste Defizite $\mathrm{zu}$ entdecken und sie besitzen hierfür möglicherweise keine ausreichende Sensitivität. Abbildung 1 veranschaulicht ein Modell zur Erklärung von Perioden mit weitgehend reversiblen Leistungsstörungen bei einer Epilepsie.

\section{Medikamente und Komorbidität}

Die präoperative Untersuchung sollte unter der für den Patienten üblichen Medikation stattfinden, also noch bevor die Dosisreduktion zur Anfallsprovokation für das Video-EEG-Monitoring erfolgt. Bei Patienten mit hohen Dosierungen in einer Polytherapie sind negative kognitive Nebenwirkungen zu berücksichtigen. Dabei sollen die „neueren“ Präparate generell unproblematischer sein als die „alten“, mit der Ausnahme von Topiramat, bei dem es in hoher Dosierung Belege für eine spezifische negative Wirkung auf Frontalhirnfunktionen gibt (9).

Die häufigste Komorbidität einer Epilepsie ist eine Depression, die in manchen Fällen auch das neuropsychologische Profil deutlich beeinflussen kann. In epilepsiechirurgischen Untersuchungsgruppen leidet 


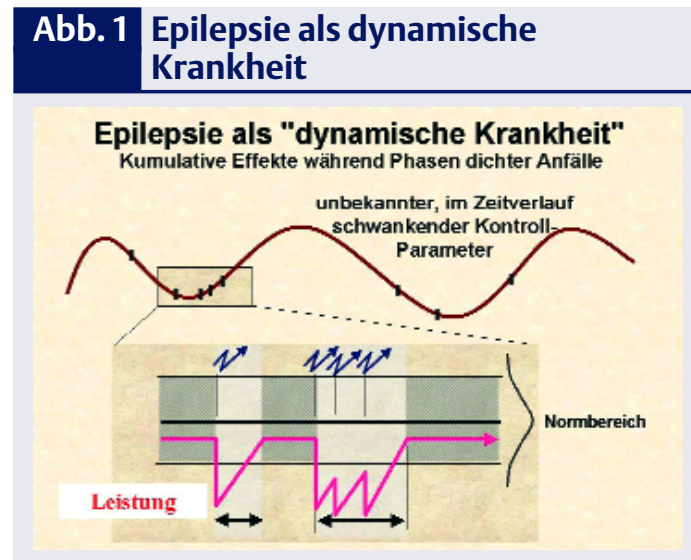

In einem komplexen System können sich durch die Verschiebung eines Parameters zu einem vom Normalen abweichenden Wert zyklische Schwankungen jener Parameter ergeben, die mit der Aktivität der Epilepsie verknüpft sind. So können Perioden mit dichten Anfällen entstehen, die von deutlichen, jedoch weitgehend reversiblen Leistungsminderungen begleitet werden (3).

jeder Dritte der Patienten an einer behandlungsbedürftigen Depression. Meistens sind diese Depressionen noch unbehandelt, u.a. auch weil pro-konvulsive Effekte einiger Antidepressiva befürchtet werden. Insbesondere unter Patienten mit einer linksseitigen TLE mit einer neokortikalen Pathologie kommt eine Depression häufig vor und ist bei diesen Patienten auch mit Gedächtnis- und Aufmerksamkeitsdefiziten verknüpft. Für diesen Zusammenhang wird eine sekundäre Beteiligung des Frontallappens bei den temporo-lateralen Epilepsien diskutiert. In den meisten Fällen sind die neuropsychologischen Störungen jedoch nicht mit der Depression verknüpft, sondern auf die fokale Epilepsie zurückzuführen.

\section{Neuropsychologische Effekte von Anfällen}

Obwohl die medikamentöse Behandlung zunächst Anfallsfreiheit anstrebt, wird dieses Ziel in ca. 40\% aller Epilepsien - insbesondere aber bei fokalen Epilepsien - nicht erreicht. In diesen Fällen muss die Therapie unterhalb dieses Zieles optimiert werden. Unter einer relativen Anfallskontrolle werden dann weitere Behandlungsgesichtspunkte wie die Verträglichkeit, die Minimierung der kognitiven Nebenwirkungen so- wie die Compliance berücksichtigt. Für eine Metaanalyse (5) wurden Längsschnitt-Studien ausgewählt, deren Behandlungen sich im Untersuchungszeitraum nicht mehr verändert haben und deren Patienten zu Beginn und am Ende des Untersuchungszeitraumes einer formalen neuropsychologischen Leistungsuntersuchung unterzogen worden waren. Für Kinder fanden sich neun Studien, in denen ausschließlich globale Leistungsmaße in der Form verschiedener Intelligenztests eingesetzt worden sind (Binet, Stanford-Binet, WISC). Darunter war eine Studie, bei denen die Kinder im dreijährigen Untersuchungszeitraum nur sehr wenige Anfälle hatten. Zwischen den Untersuchungen im Alter von vier und sieben Jahren zeigten sich hier keine Verluste in den Intelligenzwerten. In allen übrigen Untersuchungen wurden Anzeichen für leichte Verluste (mehr als 2 IQ Punkte) beobachtet, die in jeder Studie die Anzahl der leichten Zugewinne überstiegen. In der Hälfte der Studien wurde jedoch auch über deutliche Verluste von mehr als 10 IQ Punkten berichtet, von denen 10-25\% der Kinder betroffen waren. Es kommt also über die Zeit zu substantiellen kognitiven Leistungseinbußen zumindest bei einem Teil der Kinder, bei denen weiterhin Anfälle bestehen. Die Konklusivität der Analyse ist jedoch eingeschränkt, weil Art und Anzahl der Anfälle in den Studien nicht expliziert wurden und so keine Korrelation der Verluste zu diesen Parametern hergestellt werden konnte. Darüber hinaus sollten auch bei Kindern die kognitiven Veränderungen mit spezifischeren neuropsychologischen Leistungsmaßen vorgenommen werden und über längere Intervalle erfolgen, als über die üblichen ein bis vier Jahre.

Für Jugendliche und Erwachsene ließen sich dreizehn Studien mit verschiedenen neuropsychologischen Leistungsmaßen finden. Dabei war in fünf Studien ein deutlich negativer Einfluss der Anfälle zu erkennen, während vier Studien gemischte oder nicht eindeutige Ergebnisse zeigten. In zwei Studien wurden keine Veränderungen in den mentalen Fähigkeiten beobachtet.
Insgesamt ist bei Erwachsenen eher von einem schwachen Zusammenhang von Anfällen und überdauernden kognitiven Einbußen auszugehen. Generell werden tonisch-klonische Anfälle dabei als schädlicher eingestuft, besonders wenn sie in Serien oder als Status auftreten. Aber auch in den vorliegenden Erwachsenenstudien waren die Angaben über Frequenz und Art der Anfälle nicht ausreichend. Nach wie vor fehlen prospektive Langzeitverläufe mit alters- und bildungsgleichen Kontrollpersonen, die den Zusammenhang von Anfällen und kognitiven Fähigkeiten aufklären.

\section{Effekte epileptoformer \\ Entladungen im EEG}

Neben den epileptischen Anfällen mit ihrem charakteristischen Anfallsmuster im EEG bestehen bei einer Epilepsie häufig auch eingestreute Entladungen zwischen den Anfällen. Dabei handelt es sich um sehr kurzzeitige, meist nur wenige Sekunden dauernde epileptoforme EEG-Veränderungen (eEEGV), die bei einem Patienten mehr oder weniger dicht vorkommen können. Die Frage, ob alleine schon solche epileptoforme EEG-Entladungen einen Einfluss auf kognitive Funktionen haben, ist gegenwärtig eingebettet in eine Diskussion über die Abgrenzung von verhaltenswirksamen EEG-Entladungen und epileptischen Anfällen. Weil die Entladungen sehr kurzzeitig sind, konvergieren die untersuchten neuropsychologischen Funktionen meist auf die Untersuchung des Reaktionsverhaltens (Reaktionszeiten, Trefferraten u.ä.). Manche Autoren betrachten solche verhaltenswirksame EEGEntladungen nicht als eine eigenständige Entität, sondern als kurzzeitige epileptische Anfälle, da sie gerade in den kognitiven Effekten, die für einen Anfall geforderten klinische Symptome sehen.

Finden die kognitiven Störungen ausschließlich während einer Episode von epileptoformen EEG-Entladungen statt und finden sich dabei keine weiteren klinischen Zeichen, so werden sie auch als „Transient Cognitive Impairments (TCI) bezeichnet. Alle anfalls-induzierten kognitiven Störungen gehören defi- 
nitionsgemäß nicht zu den TCI. Der Nachweis von TCI erfordert im Einzelfall einen beträchtlichen apparativen Aufwand u.a. ein digitales EEG mit synchroner Videoaufzeichnung und einer computergesteuerten Aufgabendarbietung. In einer Stichprobe von über tausend Routine-EEG-Untersuchungen fanden sich TCI jedoch nur bei 2,2\% der Patienten (2).

Ein weiteres methodisches Problem im Nachweis der Verhaltenswirksamkeit kurzzeitiger epileptoformer EEG-Veränderungen ergibt sich aus der Tatsache, dass sie in ihrem Auftreten deutlich an Vigilanzabsenkungen geknüpft sind. Will man die Verhaltenswirksamkeit bei einem Patienten nachweisen und gibt man ihm hierzu eine anspruchsvolle Reaktionsaufgabe vor, so kann es vorkommen, dass der Proband dadurch eine starke Vigilanzsteigerung erfährt und als Konsequenz im EEG die kritischen Veränderungen vollständig unterdrückt werden. Aus dieser Sicht ist es keineswegs klar, ob die TCI nicht selbst Ausdruck kurzzeitiger Vigilanzeinbrüche sind.

\section{Delayed Response Aufgaben}

Eine pragmatische Methode zum Nachweis der Verhaltenswirksamkeit kurzzeitiger EEG-Veränderungen sind „Delayed Response“ Aufgaben, die jedoch nicht klären, ob es sich bei den EEG-Veränderungen um kurzzeitige Anfälle oder um TCI handelt (Abb. 2). Von einem Computer werden z.B. aus zehn möglichen Figuren zwei zufällig ausgewählt, die im Abstand von mehreren Sekunden auf dem Bildschirm kurzzeitig dargeboten werden. In zufälliger Abfolge wird in 50\% der Fälle jedoch zweimal die gleiche Figur gezeigt. Der Patient soll bei jedem Durchgang entscheiden, ob die beiden gezeigten Figuren identisch waren oder nicht. Um den Vergleich richtig vornehmen zu können, muss der Patient die erste Figur solange im Kurzzeitgedächtnis halten, bis die zweite Figur erscheint. Immer wenn eine Figur präsentiert wird, wird auch eine Markierung auf einem EEG-Kanal gesetzt. Nach ca. 100 Durchgängen wird das EEG daraufhin analysiert, bei welchen Durchgängen sich im KZG-Behaltensintervall eine EEG-Entladung ereignete und bei welchen nicht. Unter der Annahme, dass eine EEG-Entladung das KZG stört, müssten die Antworten mit EEG-Entladungen im Behaltensintervall langsamer und fehlerhafter sein als die Antworten bei den Durchgängen ohne Entladungen. Das kann durch einen statistischen Test entschieden werden. Bei seltenen EEG-Entladungen kann die Anzahl der Durchgänge erhöht werden. Ist das KZG des Patienten ohnehin schon schlecht, kann man das Behaltensintervall verkürzen.

\section{EEG-Veränderungen und subtile non-konvulsive Anfälle}

In vier Gruppenstudien mit Patienten, die häufig epileptoforme EEG-Entladungen hatten, ließ sich kein Zusammenhang zwischen kognitiven Störungen und epileptoformen EEG-Entladungen finden. In einer weiteren Studie wurde die kognitive Leistungsfähigkeit von drei Patientengruppen und einer Kontrollgruppe verglichen. Eine Gruppe hatte während der kognitiven Untersuchung weder eEEGV noch subtile non-konvulsive Anfälle ( $s n A$ ), eine hatte eEEGV aber keine snA und eine hatte sowohl eEEGV als auch snA. In Übereinstimmung mit den vorherigen Ergebnissen zeigte die Gruppe mit den snA die niedrigsten Leistungen in den kognitiven Tests. In Abwesenheit von snA schienen die epileptoformen EEG-Entladungen in dieser Studie keinen unabhängigen Effekt auf die kognitiven Funktionen zu haben. Lediglich in einer Studie konnten durch eine große Stichprobe und den Einsatz multivariater statistischer Methoden milde Effekte der epileptoformen EEG-Entladungen auf die kognitiven Leistungen bei einer einmaligen Untersuchung nachgewiesen werden. Verlaufsuntersuchung zeigen jedoch, dass es bei Patienten mit größerer Entladungsdichte über die Zeit zu einem Verlust von IQ Punkten kommt, ähnlich wie bei Patienten mit häufigen subtilen non-konvulsiven Anfällen. Perioden mit einer großen Dichte von epileptoformen Entladungen können bei Kindern zu einem Entwicklungsstill-

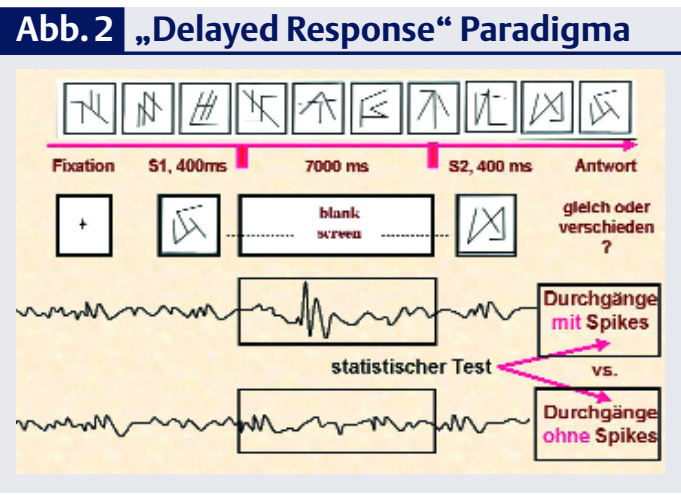

stand oder sogar zur Verhaltensregression führen, so dass einige Autoren von kumulativen Effekten ausgehen.

Im Zusammenhang mit regionalen und generalisierten Hirn-Reifungsstörungen kommt es vigilanzabhängig zu vermehrten Entladungen, so beim „electrical status epilepticus during slow wave sleep“ (ESES) und dem Landau-Kleffner Syndrom (LKS), das durch eine Regression der lautsprachlichen Entwicklung gekennzeichnet ist (verbal auditory agnosia). Obwohl auch hier die Leistungs- und Verhaltensstörungen mit den Perioden der maximalen Entladungsdichte im EEG variieren, geht man davon aus, dass sie nur milde eigenständige Effekte haben. Die epileptoformen EEG-Entladungen werden als Epiphänome betrachtet, welche ebenso wie die neuropsychologischen Leistungsstörungen durch die zu Grunde liegende Hirnpathologie verursacht werden.

Beim Syndrom der autistischen Regression zeigen sich häufig ähnliche EEG-Abnormalitäten wie beim ESES und LKS und die Verbesserung des kognitiven Status nach dem Beginn einer medikamentösen Behandlung wird oft als Beleg für die negativen Effekte der EEG-Korrelate angesehen. Diese Einschätzung stützt sich jedoch auf Einzelfälle und ist insgesamt nicht völlig überzeugend. Eine Zusammenfassung über Verhalten und Kognition bei pädiatrischen Epilepsien findet sich in (4).

Beim nonkonvulsiven Status epilepticus (NCSE) unterscheidet man eine generalisierte Form (GNSE, absence status) und eine fokale Form (complex partial status epilepticus, 
CPSE). In mehreren Verlaufsuntersuchungen mit großen Fallzahlen wurden nur in vier Fällen Gedächtnisstörungen beobachtet, die über Wochen anhielten. Daraus wurde die Schlussfolgerung gezogen, dass bei NCSE nicht mit Langzeitfolgen in der Form von kognitiven Störungen zu rechnen sei. Diese Einschätzung wird aber kontrovers diskutiert, da Tierexperimente Zelluntergänge im Hippokampus bei NCSE nachgewiesen haben. Die wesentlichste methodische Kritik an allen vorliegenden Studien ergibt sich jedoch daraus, dass nur in fünf von insgesamt 229 berichteten Fällen eine ausreichende Ausgangsuntersuchung aus der Zeit vor dem Status vorlag, zu der die Leistungs- und Verhaltensänderungen durch den NCSE in Beziehung gesetzt werden konnten.

In einer Studie wurden Patienten untersucht, die einerseits durch kognitive Leistungsfluktuationen aufgefallen waren und bei denen schwer erkennbare kurzzeitige Anfälle bestanden. Überraschenderweise kamen kurze Anfälle mit einem fokalen Beginn dreimal so häufig vor wie Absencen.

Als kritische Zeitdauer, ab der epileptoforme EEG-Entladungen leistungs- bzw. verhaltenswirksam sind, hat sich in den Untersuchungen eine Grenze um drei Sekunden herausgebildet. Hält eine epileptoforme EEG-Entladung länger an als drei Sekunden, so finden sich meistens weitere iktuale Phänomene und es findet ein (kurzzeitiger) Anfall statt. Bei kürzeren Zeitspannen war es nicht möglich, die EEG-Episoden mit kognitiven Störungen zu korrelieren oder es bestanden überhaupt keine kognitiven Effekte. Zum Nachweis der Verhaltenswirksamkeit haben sich neben Reaktionszeiten insbesondere visuelle Suchaufgaben bewährt. Aufgaben unter visueller Darbietung, die kognitiv anspruchsvoll sind und lange dauern, scheinen besonders geeignet zu sein.

Wenn der Nachweis geführt werden kann, dass bei einem Patienten die epileptoformen Entladungen verhaltenswirksam sind, bietet das die Grundlage für eine medikamentöse Behandlung. Die weitere klinische Bedeutung aus dem Nach- weis der Verhaltenswirksamkeit ergibt sich aus der Möglichkeit akkumulierende Effekte zu verhindern. Durch eine Behandlung kann bei Kindern in diesen Fällen ein nachteiliger Einfluss auf die mentale und schulische Entwicklung unterbunden werden. Aus Tierexperimenten ist bekannt, dass epileptoforme EEG-Entladungen bei der Gedächtnisbildung die Langzeit-Potenzierung unterbrechen und so eine neurophysiologische Erklärung für die akkumulierenden Effekte abgeben.

\section{Verursachen Anfälle auch bleibende kognitive Defizite?}

Ein konvulsiver Status epilepticus (epileptische Aktivität $30 \mathrm{~min}$ ) stellt eine schwere Krise dar und kann zu diffusen und lokalen Hirnschädigungen führen. Darüber hinaus belegen tierexperimentelle Untersuchungen, dass sowohl prolongierte als auch kurze Anfälle irreversible kognitive Störungen verursachen können (8). Die Empfindlichkeit für überdauernde Verhaltensstörungen wird bei Tieren durch eine Reihe von Faktoren beeinflusst. Hierzu gehören genetische Faktoren, Entwicklungsstand des Gehirns, Ausmaß und Lokalisation der Hirnläsion, Anzahl und Dauer der Anfälle sowie Reservekapazitäten des Gehirns. Günstige soziale Lebensbedingungen und eine interessante und anregende Umgebung haben protektive Einflüsse. Als Hauptmechanismus werden neurodegenerative Prozesse in den vulnerablen Zellen des Hippokampus und der Amygdala angesehen. Daneben stört epileptische Aktivität unmittelbar die synaptischen Prozesse, die zur Konsolidierung von Gedächtnisinhalten erforderlich sind. Dies zeigt sich in verstärkten Defiziten, sobald bei den Tieren die Verhaltenstests in zeitlicher Nähe zum letzten Anfall vorgenommen werden.

Besides the recurring seizures a patient with epilepsy is often confronted with impairments of his cognitve functions. Individual patients may find these cognitive consequences even more debilitating than the seizure itself. Mostly the patients complain about a general slowing of their mental processes, about difficulties with tasks in which complex information processing is needed or about impairments of memory, attention and concentration. In each case a number of epileptological factors determins the profile of cognitive impairments. Clinical neuropsychology contributes to the assessment, preservation or even the improvement of cognitive function in epilepsy patients. It makes the treatment of epilepsies saver and it has meanwhile developed to an indespensable tool to control for the out-come quality for epilepsy treatments - even beyond the question of seizure control.

\section{KeyWords:}

cognitive impairment - clinical neuropsychology - transient cognitive impairment - epilepsy surgery - memory impairment

\section{Literatur}

1. Aldenkamp AP, Baker GA, Meador KJ. The neuropsychology of Epilepsy: what are the factors involved? Epilepsy \& Behavior 2004; 5 (Suppl 1): 3-13

2. Aldenkamp AP, Arends J. Effects of epileptoform EEG discharges on cognitve function: Is the concept of „transient cognitve impairment" still valid"? Epilepsy \& Behavior 2004; 5 (Suppl 1): 25-34

3. an der Heiden U. Mathematische Grundlagen der Medizin. Medizinische Klinik 1998; 93: 557-564

4. Besag FMC. Behavioural aspects of pediatric epilepsy syndromes. Epilepsy \& Behavior 2004; 5 (Suppl 1): 3-13

5. Dodrill CB. Neuropsychological effects of seizures. Epilepsy \& Behavior 2004; 5 (Suppl 1): 21-24

6. Helmstaedter C. Neuropsychological aspects of epilepsy surgery. \& Behavior 2004; 5 (Suppl 1): 45-55

7. Jokeit H, Schacher M. Neuropsychological aspects of type of epilepsy and etiological factors in adults. Epilepsy \& Behavior 2004; 5 (Suppl 1): 14-20

8. Majak K, Pitkänen A. Do seizures cause irreversible cognitive damage? Evidence from animal studies. Epilepsy \& Behavior 2004; 5 (Suppl 1): 35-44

9. Ortinski P, Meador KJ. Cognitive side effects of antiepileptic drugs. Epilepsy \& Behavior 2004; 5 (Suppl 1): 60-65

\footnotetext{
Korrespondenzadresse:

Dipl.-Psych. Heinz Hättig

Epilepsie-Zentrum Berlin-Brandenburg im Verbund der v. Bodelschwinghschen Anstalten Bethel

Evangelisches Krankenhaus

Königin Elisabeth Herzberge

Akademisches Lehrkrankenhaus der Charité Herzbergstr. 79

10365 Berlin

h.haettig@keh-berlin.de
} 